\title{
Wandering Spleen-Case Report.
}

\author{
Tabowei B. I., Kombo BB, \\ MBBS, FMCS consultant surgeon, Niger Delta University Teaching Hospital, Okolobiri, Bayelsa state. \\ MBBS, FRCS. Consultant Surgeon, University of Port Harcourt Teaching Hospital, Port Harcourt.
}

\begin{abstract}
Wandering spleen is a rare clinical entity. It can be complicated by torsion which is a surgical abdominal emergency. We report a case of wandering spleen which presented as an acute abdomen. Patient had laparotomy and did well postoperatively.

Key Words: Wandering Spleen, splenic torsion, splenectomy.
\end{abstract}

\section{Introduction}

Wandering spleen is a rare condition with a constant danger of splenic torsion. 1-25. This may lead to splenomegaly, splenic infarction, varicose and heamorrhage.1-3

The etiology is multifactorial and includes both congenital and acquired causes. (13)

It mainly occurs consequent to an embryonic disturbance in the development of the ligament connecting the spleen with the surrounding tissues $.3,4,6,9,13$,. Its incidence ranges between $0.2-0.5 \%$. $(22,23$,$) and it occurs$ 15 times more often in women than men, especially in women between the ages of 20-40 years, suggesting a hormonal factor.(1,2, 7,8) The spleen can be found anywhere in the abdomen or the pelvic region depending on the length of its pedicle. (1-27) This predisposes it to rupture, infection, or trauma. 9, 10,

It is often difficult to make a clinical diagnosis because there is no unspecific clinical manifestation.(127) $60 \%$ of patient s experiments abdominal pain and discomfort, while the rest are asymptomatic. Therefore, a high index of suspicion is required by clinicians to make a diagnosis.(1-27)

Laboratory findings are non-specific and nod-diagnostic. These includes leucocytosis, thrombocytopenia and anemia.(1)

In this environment, ultrasonography is the standard investigation for a diagnosis. However, where available, CT Scan, MRI, angiography, Scintilography and Doppler scanning are used to confirm the diagnosis. (3 23,24, 25, 26, 27.)

Treatment is usually controversial (12), but splenectomy should be considered in cases of non-viability of the spleen. Splenopexy is for those with viable spleen and laparoscopic splenopexy is very useful in places where it is available. $(23,24,25,26$. $)$

In children, splenic preservation is always preferred in order to minimize the risk of infection especially with encapsulated organisms such as pneumococcal infection. (14) Torsion from its long pedicle is the most common complication. It presents as an acute abdominal emergency. This is sometimes associated with other manifestation like gastric or pancreatic tail volvulus which can lead to acute pancreatitis and its sequelae $(1,2,3,-22)$

\section{Case Report}

A.B a 15 year old girl presented with recurrent attacks of abdominal pain over a 4 years duration. The pain was throbbing in nature, associated with nausea and sometimes vomiting. The current episode leading to her hospitalization had been persistent and associated with abdominal distention. She denied a history of trauma, constipation and urinary symptoms. She was nulliparous.

Examination revealed a young girl in painful distress, not pale, anicteric and afebrile to touch.

Pulse was $100 / \mathrm{min}$, Blood pressure $100 / 60 \mathrm{mmhg}$; temperature $-37.6^{\circ} \mathrm{C}$.She had mild abdominal distension with generalized abdominal tenderness.

A mobile and tender mass of 15 by $10 \mathrm{~cm}$ was felt at the pelvic region. Rectal examination done revealed nothing significant. The Urea and electrolytes, Creatinine, Full blood count, and urinalysis were done and all were within normal range.

Abdominal ultrasound revealed an empty splenic region, but a homogenous hypo-echoic mass measuring 10 by 15 by $8 \mathrm{~cm}$ in the lower abdomen suggestive of an ectopic spleen.

At laparotomy, the spleen was located at the pelvic region, with a long pedicle. There were enlarged dilated tortuous veins(varices) surrounding the pedicle of the spleen and the greater curvature of the stomach.

The pedicle was twisted, but the spleen looked normal and viable, but it had no notch. 
Splenectomy was performed because of difficulty in relocating the spleen and the portal hypertension the spleen was causing. She had an uneventful postoperative recovery and was discharged home on the $9^{\text {th }}$ post operative day. She was placed on long term Ampiclox and Paludrine prophylaxes.

\section{Discussion. \\ Wandering spleen is the displacement of the spleen from its normal location due to loss or weakening of the ligament of the spleen. $(5,6)$. \\ It was first described by Von Horne in 1667 during an autopsy (25), and it is characterized by a lax} ligamentous attachment of the spleen to the diaphragm, colon, and the retro-peritoneum forming a mesospleen. $(6,7,9,10)$

This rare clinical entity is caused mainly by a congenital failure of the dorsal mesogastrum to develop when the lesser sac is formed.(i)

The acquired form occurs mostly in multiparous females as the ligaments which are holding the spleen in position become lax $(1,22,25$,$) . This has been attributed to hormonal changes, increase in spleen size, and a$ weakness of the abdominal wall $(16,18,19,20,27$,$) .$

It is 15 times more common in females than males suggesting a hormonal influence as was demonstrated in this case. $(1,1618,20,19,27$,$) .$

The incidence based on several large series of splenectomies ranges between 0.2-0.5\%.(1,2). In our environment, no data is available, but with the rarity it could be put at $0.2 \% .(7,24,25)$

Its clinical presentation is vague and variable (1-27). 60\% of the patients experience acute or chronic recurrent abdominal pain and discomfort while the rest are asymptomatic and incident finding at autopsy.(1,17,22,)

The index case had chronic abdominal pain which has lasted more than four years. This tends to agree with the earlier reported cases.

The haematological and biochemical investigations results are usually non-specific (2) as was demonstrated in this case. The main diagnostic standard in our environment is the non-invasive procedures of ultrasonography.(2) CT an and MRI are other useful investigative tools which also include angiography, and scintigraphy.(23-27)

However, in our environment ultrasonography scan is still considered the most reliable tool for making a diagnosis. It was also used in this case where the scan showed the empty splenic fossa, and located the spleen in the pelvic region. Besides it being available, it is cheap, non-invasive and easy to operate. Its specificity is high, and it has no known recorded side effect.

Non-operative treatment of a wandering spleen is associated with a complication rate as high as $65 \%$ in some series,(1,6,17.8). Splenectomy and splenopexy are the accepted treatment options depending on viability of the spleen and the surgeons ability to do a splenopexy at its normal site or its new site.

Splenic preservation would have been preferred $(1,17)$ but our patient had splenectomy as a result of the long period of symptoms, splenic long pedicle and its position in the pelvis, presence of adhesions and varices in the stomach and the abdomen.

Postsplenectomy sepsis especially with encapsulated organism may occur with a mortality of $30-60 \% .(17,28)$ As a result the patient was placed on long time prophylactic antibiotic and anti-malarial treatment. Therefore splenopexy is advocated in the uncomplicated case with a viable spleen.

The best treatment option today is laparoscopic splenopexy $(17,28,29)$. This is advocated if the knowledge, equipment and technical know-how are available.

Acute gastric hemorrhage secondary to wandering spleen has been reported by Angeras et al(30). In this case, a peduncular torsion resulted in impaired blood return via the splenic veins. As a result, collateral circulation then built up through the short gastric vessels leading to the formation of isolated gastric varices and ultimately bleeding from the stomach fundus. The presence of varices in both the pedicle and stomach in our case was similar to what was reported by Angeras et al. but in our case, there was no bleeding.

In children splenic preservation is always preferred in order to minimize the risk of infection (14)especially with encapsulated organisms. Patients who have had splenectomy should be followed up for a long time and some be immunized against these organisms. In the presence of any infection, aggressive antibiotic therapy should be instituted

\section{Conclusion}

We have reported a case of a wandering spleen which to the best of our knowledge has not been reported in this region and reviewed the literature. Early diagnosis and splenopexy is recommended especially in women of child bearing age. This is to rule out splenic rupture during pregnancy and eliminate the chances of volvulus of its long pedicle. 

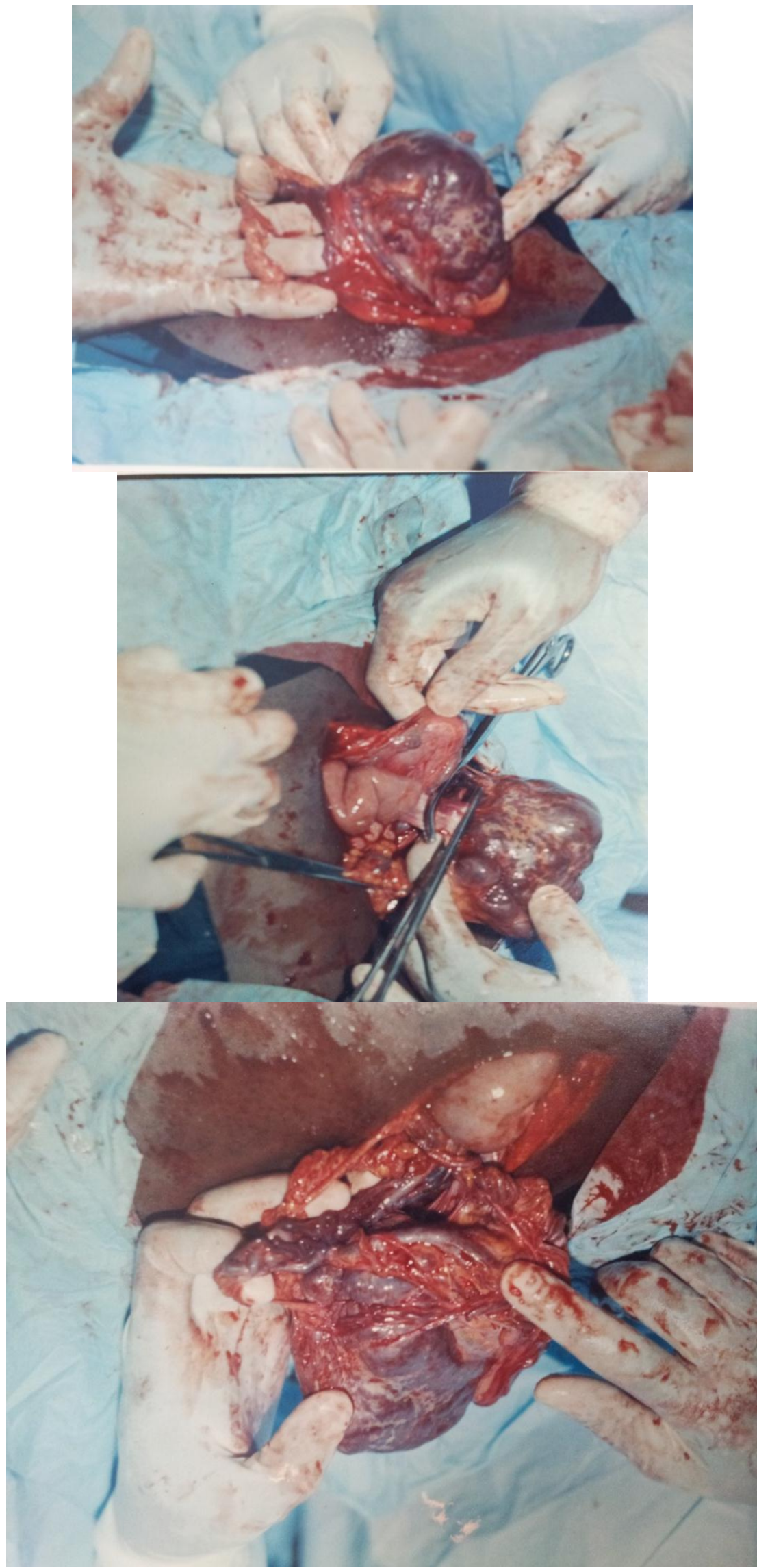


\section{References}

[1]. Desei DC, Hebra A, Davidoff AM, et al. wandering spleen: a challenging diagnosis. South Med. J 1997; 90: 439-43

[2]. Ugwu AC, Ogbonna CO et al. A wandering: A common presentation of an uncommon anomaly. SA Fam pract, 2010; 52(1): 4243.

[3]. Faisia M. Al-Mashat, Abdulrahman M.Sibiany et al. Wandering spleen. Saudi Med J 2004; Vol.25 (1): $91-94$.

[4]. E Broadis, M K Banda et al. paediatric wandering spleen in Malawi. Malawi Medical Journal ;2010; 22 (4): $120-121$.

[5]. Sheflin JR, Lee CM, Kretchmar KA. Torsion of wandering spleen and distal. AJR Am J Roentgenol 1984; 142: 100-1.

[6]. Eraklis AJ, Filer RM. Splenectomy in childhood: a review of 1413 cases. J pediatric surg 1972; 7: 382-8.

[7]. Ferandex EM, Gonzales IA, Malagon AM,et al. An unusual case of hemoperitoneum owing to acute splenic torsion in a child with immoglobulin deficiency. J Postgrad Med 2006; 52: 42-42.

[8]. Singh I. essential of anatomy. New Delhi. Jaypee Brothers medical Publishers Ltd; 2002. P283.

[9]. Andley M S, Chibber P, Ravi B, kumar A. Internal herniation of wandering spleen; a rare cause of recurrent abdominal pain. Int Surg 2000; 322-324.

[10]. Horwitz JR, Black CT. Traumatic rupture of wandering spleen in a child: case report and literature review, J Trauma 1996; 41: 348- 350 .

[11]. Alawi MH, Khalifia A, Bana H. wandering spleen: a challenging diagnosis. Park J Med Sci 2005; 21(4) $482-4$.

[12]. Gurski RR, Schirmer CC, Fischer CA et al, Laparoscopic approach to wandering spleen: a case report and update to the question. Surg Laparosc Endosc 1998; 8 363-365.

[13]. Thomson JS, Ross RJ, Pizzaro ST. The wandering spleen in infancy and childhood. Clin pediatr(Phila)1980; 19221 - 224.

[14]. Dalpe C, Cunningham M. Wandering spleen as an asymptomatic pelvic mass. Obstet Gynecol 2003; 101(5pt2): $1102-1104$.

[15]. Sucandy I, Akmal, Gabrielsen JD,. Spontaneous massive hemoperitoneum: A potentially life threatening presentation of the wandering spleen. North Am J Med Sci, 2011; 3: 99-102

[16]. Koppilkar MG, Vaze AM Bapat RD. wandering spleen. J Postgrad Med. 1981;27: 42.

[17]. Maxall-Armstrong C A , E D V Clark, T M Tsang, RJ Stewart, The wandering spleen, Archives of disease in childhood 1996, 74; 247- 248

[18]. Allen KB, Gay BB, Skandalakakis JE. Wandering spleen: Anatomical and radiological consideration. South Med J 1992; 85: 976984.

[19]. Stringel G, Soucy P, Mercer S. Torsion of the wandering spleen: splenectomy or splenopexy. J Pediatr Surg 1982; 17: 373-5.

[20]. Robinson AP wandering spleen: case report and review. Mt Sinai J Med 1988; 55(5): 428-434.

[21]. Dawson JH, Roberts NG. management of the wandering spleen. Aust N Z J surg !994, 64(6); 441-444.

[22]. Buchner M, Baker MS. The wandering spleen. Surg gynecol obstet 1992; 175 373-387.

[23]. Ben Ely A, Seguier E, Lotan G, et al. Familial wandering Spleen: a first instance. J pediatr Surg 2008; 43: E23-5

[24]. Kinori I, Rifkin MD, A truly wandering spleen. J ultrasound Med. 1988; 7 :101-5.

[25]. Fujiwara T, Takehara Y, Isoda $\mathrm{H}$ et al, Torsion of the wandering spleen: CT and angiographic appearances. J comput Assist Tomogr 1995; 19 84-6.

[26]. Danaci M, Belet U, Yalin T, Polat v et al, Power Doppler sonographic diagnosis of torsion in a wandering spleen. $\mathrm{J}$ clin Ultrasound 2000; 28: 246- 248 .

[27]. Befikadu S, Gudu W, Absenno N, Torsion of a pelvic wandering spleen as a cause of acute abdomen in a woman : case report and review of the literature. Ethiop Med J 2004; 42: 53-61.

[28]. Tan HH, Ooi LLPJ, Tan CK. Recurrent abdomoinal pain in a woman with a wandering spleen. Singapore Med J 2007; 48: e122-4.

[29]. Balfanz JR, Nesbit ME,JarvisC et al. Overwhelming sepsis following splenectomy for trauma. J Pediatr 1976;88: 458-9

[30]. Hirose R, Kitano S, Bando T, Ueda Y, Sato K, Yoshida, et al. Laparoscopic surgery for pediatric wandering spleen. J Pediatr Surg 1998; 33: 1571-1573.

[31]. Angeras U, Almskog B, Lukes P, et al. Acute gastric hemorrhage secondary to wandering spleen. Dig Dis Sci 1984; 29:11591163 . 